\title{
Optimization of camptothecin production and biomass yield from endophytic fungus Fusarium solani strain ATLOY-8
}

Prince Clarance, Ameer Khusro, Jebalsy Lalitha, Jerin Sales, Agastian Paul*

Research Department of Plant Biology and Biotechnology, Loyola College, Chennai, India.

\section{ARTICLE INFO \\ Received on: 08/02/2019 \\ Accepted on: 22/06/2019 \\ Available online: 05/10/2019}

Key words:

Biomass, Box-Behnken

design, camptothecin,

Fusarium solani,

optimization.

\begin{abstract}
Fusarium solani strain ATLOY-8, a fungus that produces camptothecin (CPT) was isolated from Chonemorpha fragrans (Moon) Alston. The process of optimization of the camptothecin yield was optimized by means of the traditional methods and analytical tools. Using the Box-Behnken design (BBD) matrix at $N=17$, three independent variables were optimized designated using one factor at a time (OFAT) method, for improved CPT and biomass production. The BBD exhibited 1.4- and 1.2-fold increase of CPT production and biomass yield, respectively, compared to OFAT approach in the basal medium containing absolute ethanol (5\%), glucose (1\%), and precursors (a combination of tryptophan, geraniol, and tryptamine; $0.03 \%$ ). Analysis of variance showed the elevated coefficient of determination $\left(R^{2}\right)$ of 0.9751 and 0.9980 , respectively, for CPT and biomass yielding at a significant level $(p>0.05)$. Three dimensional graphs revealed dependent synergy between two variables to improve the CPT and biomass yield.
\end{abstract}

\section{INTRODUCTION}

Camptothecin (CPT) is a naturally occurring alkaloid. This alkaloid has the ability to prohibit the topoisomerase I; thus, it is an anticancer compound (Hsiang et al., 1988; Kjeldsen et al., 1992; Thomas et al., 2004). CPT has derivatives such as topotecan and irinotecan which are used in the medication of colon and ovarian cancers (Demain and Vaishnav, 2011). The demand for CPT per year is approximately $3,000 \mathrm{~kg}$ in the international market and the annual production throughout the globe is only 600 $\mathrm{kg}$ which cannot meet the demand (Raveendran, 2015).

The first isolation of camptothecin is owed to the elicitation of Camptotheca acuminata in China and Nothapodytes foetida in India (Lorence and Nessler, 2004). CPT has been spotted in species of plants from unrelated classes of Asterids and Rosids, families, such as Icacinaceae (Pyrenacantha klaineana and Merrilliodendron megacarapum), Gelsemiaceae (Mostuea

\footnotetext{
${ }^{*}$ Corresponding Author

Agastian Paul, Associate Professor, Research Department of Plant Biology and Biotechnology, Loyola College, Chennai, India.

E-mail: agastianloyolacollege@gmail.com
}

brunonis), Apocynaceae (Ervatamia heyneana), Rubiaceae (Ophiorrhiza pumila), and Nyssaceae (C. acuminata) (Backlund et al., 2000; Hilu et al., 2003). Recently, the demand for CPT caused massive harvest of those trees in Asia (Shwetha et al., 2013). Under such a critical situation, there is an immediate need for finding new plant sources and alternative methods for the consistent supply of important compounds like CPT.

In this study, the authors isolated Fusarium solani from the root of the host plant Chonemorpha fragrans (Apocynaceae), which is marked as endangered in Karnataka state and vulnerable in Kerala state of India (Khan et al., 2005). The fungus is known to produce CPT in distinct media under shake flask culture. The optimum manufacture of CPT in a profitable manner can be achieved by applying alternative methods. Hence, further exploration is required to enhance extraction through varied techniques (Khusro and Aarti, 2015; Khusro et al., 2016). The optimization for dependent variables is proportionate to medium diverse factors such as carbon source, nitrogen source, $\mathrm{pH}$, temperature, agitation speed, and incubation period. One factor at a time (OFAT) based optimization is a tedious method with less accuracy. To raise the accuracy level and yield, Response Surface Methodology (RSM) is implied nowadays with high accuracy (Bocchini et al., 2002). Considering this, the contemporary context 
was scrutinized to augment the CPT production and biomass yield from a new strain of endophytic fungus segregated from $C$. fragrans using RSM tool.

\section{MATERIALS AND METHODS}

\section{Plant material}

Chonemorpha fragrans, a stout spreading laticiferous shrub was gathered from the Western Ghats of the southern region of Kerala. The mature plant part of roots was collected in a clean and dry plastic bag. The roots showed dark brown in color at the epidermal layer. The roots collected were brought to the laboratory for processing. Prof. Dr. G. Jeyajothi, Taxonomist, Loyola College, Chennai, India, identified and verified the plant. The voucher specimen (No: 314) has been retained in the Department herbarium.

\section{Isolation of endophytic fungus}

The plant parts collected were cleaned using deionized water. Surface sterilization of explants was carried out as per the methodology of Hallmann et al. (2006) with minor modifications. The root selected were washed in $0.1 \%$ Tween 20 for 30 seconds, followed by $0.1 \%$ Bevistin for 2-3 minutes to prohibit the existence of external fungi. After that, samples were saturated in $0.1 \%$ sodium hypochlorite for 30 seconds and finally in $70 \%$ $\mathrm{v} / \mathrm{v}$ ethanol for 3-5 minutes. After specific treatment, samples were rinsed thrice in sterile distilled water. After the surface sterilization process, the plant parts were finally desiccated in the laminar airflow chamber. Once the samples were dried, they were aseptically dissected to project the cortex region and placed on to aqueous agar $(1.5 \%$, HiMedia, Mumbai) medium and incubated for 4-5 days at $28^{\circ} \mathrm{C} \pm 2^{\circ} \mathrm{C}$ in dark. The isolation medium was complemented with streptomycin $(250 \mathrm{mg} / \mathrm{l})$ for the prevention and growth of bacteria. A negative control was placed using the explants without the sterilization process to check the surface contaminated fungi.

\section{Morphological and molecular characterization of fungus}

The fungal species isolated was subjected for morphological assessment according to Barnett (1998). Further, the species was characterized using molecular techniques. Primers (ITS1 and ITS 4) were used for the amplification process (White et al., 1990). The fungus was grown in PDA plate and incubated at $28^{\circ} \mathrm{C} \pm 2^{\circ} \mathrm{C}$ for 6 days. Within 6 days, white thick cottony mycelium was formed in the plate. The mycelium was taken apart from the culture. Approximately $25 \mathrm{mg}$ of the tissue/mycelium was macerated using liquid nitrogen. In a microcentrifuge tube, the granulated tissue was collected. Using this powdered tissue, DNA isolation is performed using NucleoSpin ${ }^{\circledR}$ Plant II Kit (Macherey-Nagel). Amplification of the Polymerase chain reaction (PCR) products was implemented by making use of PCR thermal cycler (GeneAmp PCR System 9700, Applied Biosystems) and universal primers. UV transilluminator (Genei) was instrumental in visualization and imaging of the PCR products and the image was photographed beneath the UV light using Gel documentation system (Bio-Rad). Total score and highest homology were noted using BLAST. The fungal sequences were deposited to Genbank, NCBI, with accession number KY497951. MEGA (Molecular
Evolution Genetic Analysis) software version 4 was used to create a phylogenetic tree.

\section{Extraction of CPT}

The fungal strain was cultured in $50 \mathrm{ml}$ of presterilized YESD (Yeast extract, Soy peptone, and Dextrose) broth (VanderMolen et al., 2013). Flasks were agitated at $180 \mathrm{rpm}$ at $28^{\circ} \mathrm{C} \pm 2^{\circ} \mathrm{C}$ for 10 days. Camptothecin was extracted from fungal mycelia using the methodology of Shweta et al. (2010) with minor adaptations. The mycelium was isolated from the culture by a cheese cloth and desiccated to moisture level $(5 \%)$ at $60^{\circ} \mathrm{C}$ for 96 hours. The desiccated mat was finely pulverized using a sterilized mortar and pestle. $0.1 \mathrm{~g}$ of powder was taken in $15 \mathrm{ml}$ vial, 10 $\mathrm{ml}$ of $61 \%$ ethanol was added and incubated overnight. After 12 hours, it was sonicated and placed at $60^{\circ} \mathrm{C}$ in shaking water bath for 3 hours. Then the essence was cooled to the room temperature, and further, the essence was subjected to centrifugation at 10,000 $\mathrm{rpm}$ (Remi, Mumbai) for 10 minutes at $10^{\circ} \mathrm{C}$. The supernatant was filtered using $0.2 \mu$ filter (Thermo fisher) and checked for CPT.

\section{Reversed-phase high-performance liquid chromatography (RP-HPLC) analysis}

Camptothecin was analyzed by RP-HPLC (LC-20AD, Shimadzu, Japan). The preparation of the typical CPT was $(95 \%$ HPLC grade Sigma Aldrich Inc USA) performed using methanol and DMSO at $3: 1(\mathrm{v} / \mathrm{v})$ ratio $(1 \mathrm{mg} / \mathrm{ml})$. Preparation of the working solution $(20 \mu \mathrm{l})$ was done $(0.1$ to $0.5 \mathrm{mg} / \mathrm{ml})$ from the stock solution and the working solution was introduced to the HPLC system to acquire the standard curve. It took 15 minutes of run time in the column. The availability of CPT was confirmed by matching with the retention time of standard. The standard CPT regression curve is noted and further quantified the CPT in the sample extract.

\section{Quadruple time-of-flight mass spectrum analysis (ESI-Q-TOF MS) analysis}

Mass spectrometry analysis was done by ESI-Q-TOF MS (Waters Micromass Q-Tof Premier Mass Spectrometer, USA) fortified with an electrospray ionization source. A syringe pump was instrumental in injecting the samples to the system at a flow rate of $10 \mathrm{ml} / \mathrm{min}$ and spray voltage was set to $1.5 \mathrm{kV}$ in positive mode.

\section{Biomass determination}

Utilizing the methodology of Khusro et al. (2017), the fungal biomass estimation was carried out.

\section{Medium optimization using OFAT method-standardization of basal medium}

Standardization of the basal medium for the optimum growth of fungi and CPT production consists of various media as given below:

Czapek Dox broth (CDB; g/l): $\quad$ NaNO3 3.0, K2HPO4 1.0, $\mathrm{MgSO} 40.5, \mathrm{KCl} 0.5, \mathrm{FeSO} 4$ 0.01 , and Sucrose 30.0

Potato dextrose broth (PDB; g/l): Potato 200.0 and Dextrose 20.0 Sabouraud dextrose broth

(SDB; g/l): YESD broth $(\mathrm{g} / \mathrm{l})$ :
Dextrose 40.0 and Peptone 10.0 Yeast extract 5, Soy peptone 20.0, and Dextrose 20.0 
Malt Extract broth (MEB; g/l): YPSS (g/l):

Malt extract 5.0

Yeast 4.0, Starch 11.4, $\mathrm{KHPO}_{4}$ 1.0, and $\mathrm{MgSO}_{4} .7 \mathrm{H}_{2} \mathrm{O} 0.5$

Peptone Yeast extract

Glucose broth (PYG; g/l):

Soy peptone 1.25 , yeast extract 1.25 , and D-glucose 5

The CPT yield and biomass produce were assessed based on the earlier described method.

\section{Effect of incubation period}

The fungal strain was inoculated into the optimum basal medium up to 14 days in shake flask condition. The CPT and biomass output were quantified according to the procedure as described earlier.

\section{Effect of ethanol concentration}

Addition of absolute ethanol is known to increase the production of CPT (Venugopalan et al., 2015). Varying ranges $(0 \%$ to $10 \%)$ of pure ethanol were administered to the suspension cultures of fungi for the estimation of CPT and biomass production as per the methodology as discussed earlier.

\section{Effect of carbon and nitrogen sources}

Dextrose, mannose, galactose, sucrose, glucose, fructose, lactose, and maltose were used for estimating CPT and biomass. These carbon sources $(1 \% \mathrm{w} / \mathrm{v})$ were supplemented into the basal medium. Effect of various nitrogen sources was examined for the biomass and CPT production of the fungi. The basal medium was individually supplemented with ammonium phosphate, ammonium sulfate, ammonium nitrate, soy peptone, beef extract, and yeast extract $(1 \% \mathrm{w} / \mathrm{v})$. The CPT production and biomass yield were assessed according to the methodology as described earlier.

\section{Effect of pH}

To determine the impact of varying $\mathrm{pH}$ on CPT and biomass yield, $\mathrm{pH}$ of basal media was adjusted up to 12.0 prior to sterilization. The CPT production and biomass yield were estimated according to the methodology as discussed earlier.

\section{Effect of temperature}

To determine the impact of temperature on the maximum yield of CPT and biomass, basal medium containing optimum medium components was incubated at different temperatures $\left(25^{\circ} \mathrm{C}-50^{\circ} \mathrm{C}\right)$ for 9 days. As per the methodology described earlier, the $\mathrm{CPT}$ production and biomass yield were estimated.

\section{Determination of agitation speed}

In shake flask culture, aeration is a central influence for the fermentation. The experiments were planned and executed by varying agitation speed ranging from 80 to $160 \mathrm{rpm}$ for seeking the optimal level following all the other standardized parameters. As per the methodology described earlier, the estimation of CPT production and biomass yield were done.

\section{Effect of precursors and elicitors}

The precursors and elicitors were supplemented to the culture on the $6^{\text {th }}$ day of the cultivation and harvested on the $9^{\text {th }}$ day. The biosynthetic precursors added were $(\mathrm{mM})$ : tryptophan
1.0, tryptamine 0.5 , and monoterpenoid alcohol geraniol 1.0. The elicitors added were $(\mathrm{mM})$ : jasmonic acid 0.25 , methyl jasmonate 0.25 , manganese chloride 0.5, BSA 0.075, and silicone oil 1.0. The estimation of CPT production and biomass yield were done according to the previously described methodology.

\section{Optimization of independent variables using RSM}

Based upon the results of the OFAT conventional methodology, Box-Behnken Design (BBD) matrix was instrumental in optimizing the most significant independent variables (ethanol, glucose, and precursors) to maximize CPT and biomass yield by keeping other variables constant. Independent variables are very important in this system. Each one of them in the design matrix was subjected for thorough study at three different levels $(-1,0,+1)$. BBD is a method of optimization of best-fit parameters using a small number of the quadratic models.

\section{Validation of model and software used}

The validation of the design was carried out by running the experiments in triplicates. Optimum values were selected. The prominent independent variables were optimized using Design Expert Version 11.0.0 (Stat-Ease Inc., Minneapolis, MN). Statistical parameters were validated by analysis of variance (ANOVA).

\section{RESULTS}

\section{Isolation of fungus}

The fungus was isolated from the root portion and showed a white cottony growth on PDA medium. The hyphae initially hyaline and mycelium turned white to yellowish; and to bluishgray after 2 weeks. The mycelium produced copious microconidia appeared to be cylindrical to oval. Macroconidia were fusiform, 3-5 septate, cylindrical, and slightly curved (Fig. 1). An amplicon of 476 bp size (Fig. 2) was observed after amplification.

\section{Identification of fungus}

The ITS rDNA data of the fungus showed $100 \%$ similarity with the existing fungal strains and identified as $F$. solani strain ATLOY-8. The sequence was submitted to GenBank, NCBI (Accession No.: KY497951). Fig. 3 shows the phylogenetic

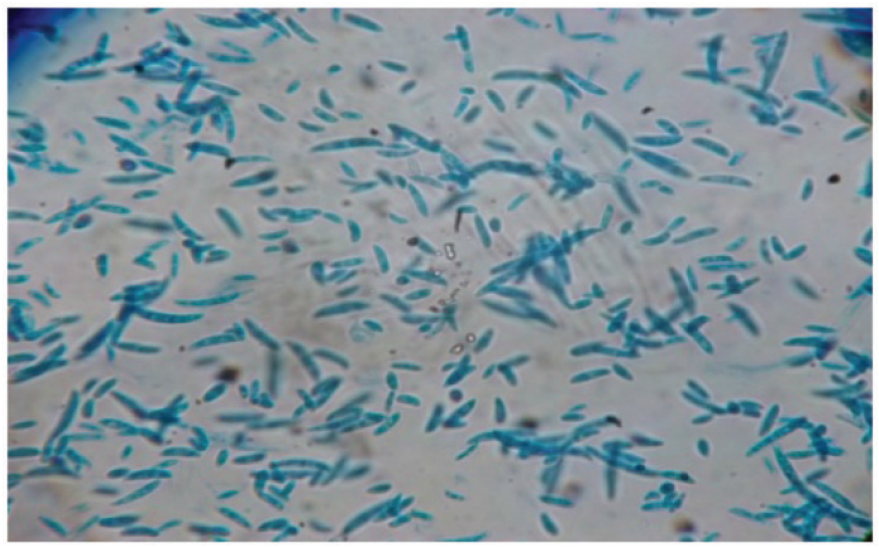

Figure 1. Septate hypha showing macroconidia and microconidia observed under Olympus $40 \times$. 
GTTGCCTCGGCGGGAACAGACGGCCCCGTAACACGGGCCGCCCCCGCCAGAGGACCCCCTAACTCTGTTT СTATAATGTTTCTTCTGAGTAAACAAGCAAATAAATTAAAACTTTCAACAACGGATCTCTTGGCTCTGGC ATCGATGAAGAACGCAGCGAAATGCGATAAGTAATGTGAATTGCAGAATTCAGTGAATCATCGAATCTTT GAACGCACATTGCGCCCGCCAGTATTCTGGCGGGCATGCCTGTTCGAGCGTCATTACAACCCTCAGGCCC CCGGGCCTGGCGTTGGGGATCGGCGGAAGCCCCCTGCGGGCACAACGCCGTCCCCCAAATACAGTGGCGG TCCCGCCGCAGCTTCCATTGCGTAGTAGCTAACACCTCGCAACTGGAGAGCGGCGCGGCCACGCCGTAAA ACACCCAACTTCTGAATGTTGACCTCGAATCAGGTAGGAATACCCGCTGAACTTAA

Figure 2. ITS rDNA sequence (476 bp) of strain ATLOY-8.

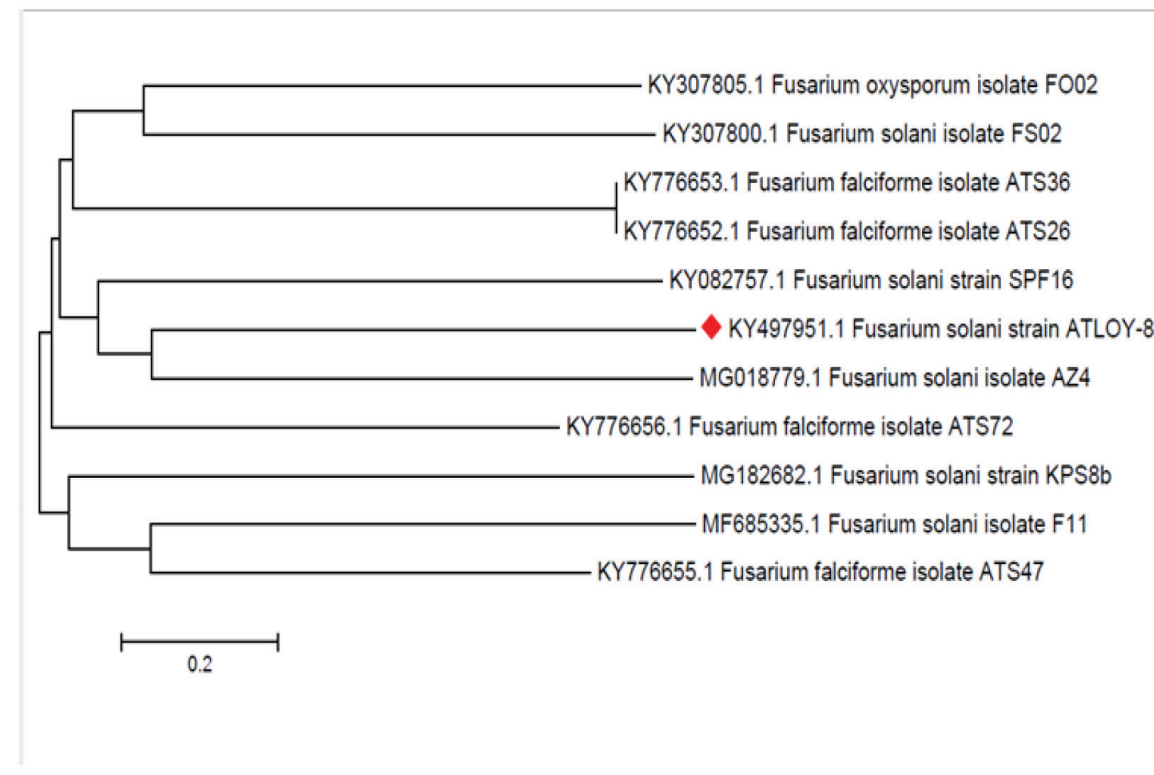

Figure 3. Neighbor-joining tree of strain ATLOY-8 showing a relationship with other $F$. solani strains based on ITS rDNA sequence data.

tree of strain ATLOY-8 constructed using bioinformatics software MEGA 4.

\section{HPLC and ESI-Q-TOF MS analysis for detection of CPT}

The crude extract of strain ATLOY-8 was analyzed using HPLC. The analysis exhibited a peak eluting at a retention time of 4.6 minutes corresponding to the standard CPT. Most of the CPT was estimated in the broth. To further confirm the presence of CPT, the partially purified extract of strain ATLOY-8 was subjected to ESI-Q-TOF MS analysis. The spectrum showed a peak at $\mathrm{m} / \mathrm{z}$ $[\mathrm{M}+\mathrm{H}]^{+} 349$ and $\mathrm{m} / \mathrm{z}[\mathrm{M}+\mathrm{H}]^{+} 379$ corresponding to the protonated molecule ions of CPT and 9-methoxycamptothecin, respectively.

\section{Effect of media}

Different media were prepared to grow and ferment strain ATLOY-8 for CPT and biomass production. The production of CPT on YESD was higher than that of PDB. The CPT content in YPSS was slightly lower than that of PDB. The CPT production was substantially reduced in CDB and MEB, respectively (Fig. 4a).

\section{Effect of incubation period}

Maximum CPT yield was obtained at 8th day of incubation while the biomass yield was increased at 9th day of incubation. The productivity of CPT and biomass yield reduced afterward (Fig. 4b).

\section{Effect of ethanol}

Addition of absolute ethanol increased the production of CPT. 5\% absolute alcohol exhibited maximum CPT production $(6.1 \pm 0.183 \mu \mathrm{g} / \mathrm{ml})$ and biomass $(14 \pm 0.42 \mathrm{~g} / \mathrm{l})$ (Fig. 4c).

\section{Effect of carbon and nitrogen sources}

Among varied carbon sources, glucose was observed to be the best inducer $(1 \% \mathrm{w} / \mathrm{v})$, followed by sucrose. Other carbon sources resulted in a reduced level of productivity (Fig. 4d).

Supplementing beef extract showed the maximum CPT yield $(7.1 \pm 0.213 \mu \mathrm{g} / \mathrm{ml})$. On the other hand, ammonium nitrate gave maximum biomass yield (16 $\pm 0.48 \mathrm{~g} / \mathrm{l})$ (Fig. 4e).

\section{Effect of pH}

The $\mathrm{pH} 5.5$ showed a maximum yield of CPT $(8 \pm$ $0.24 \mu \mathrm{g} / \mathrm{ml}$ ). However, $\mathrm{pH} 6$ gave maximum biomass yield (15 $\pm 0.45 \mathrm{~g} / \mathrm{l})$. Further, acidic $\mathrm{pH}$ reduced the growth of mycelia significantly and at alkaline $\mathrm{pH}$, the growth of mycelia was very slow and subsequently reduced the yield of both the CPT and biomass (Fig. 4f).

\section{Effect of temperature and agitation speed}

Temperature is an important physical factor for the growth of mycelia in a medium. Strain ATLOY-8 showed maximum biomass production at $25^{\circ} \mathrm{C}(17 \pm 0.51 \mathrm{~g} / 1)$. As the temperature 


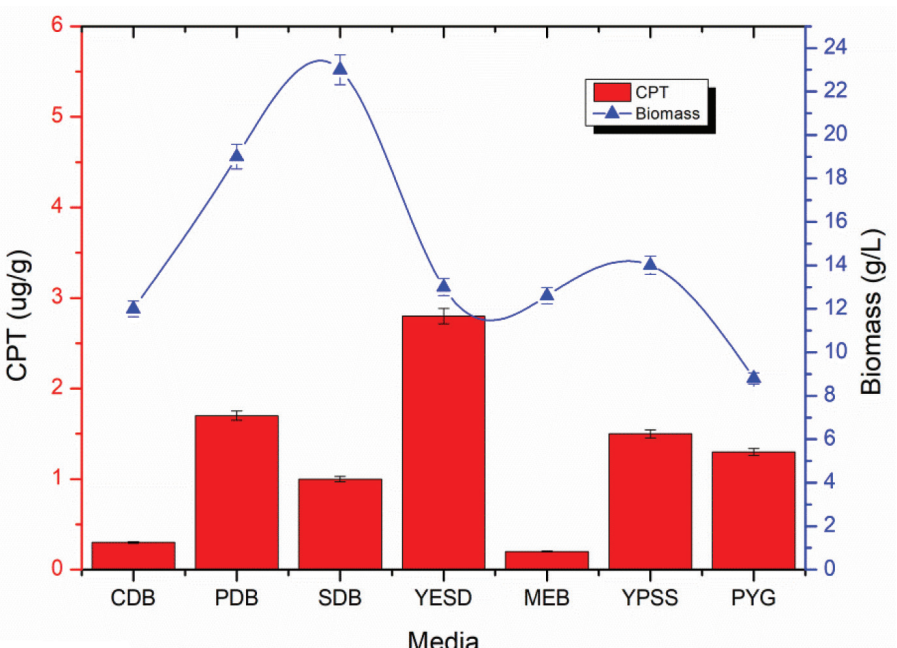

(a)

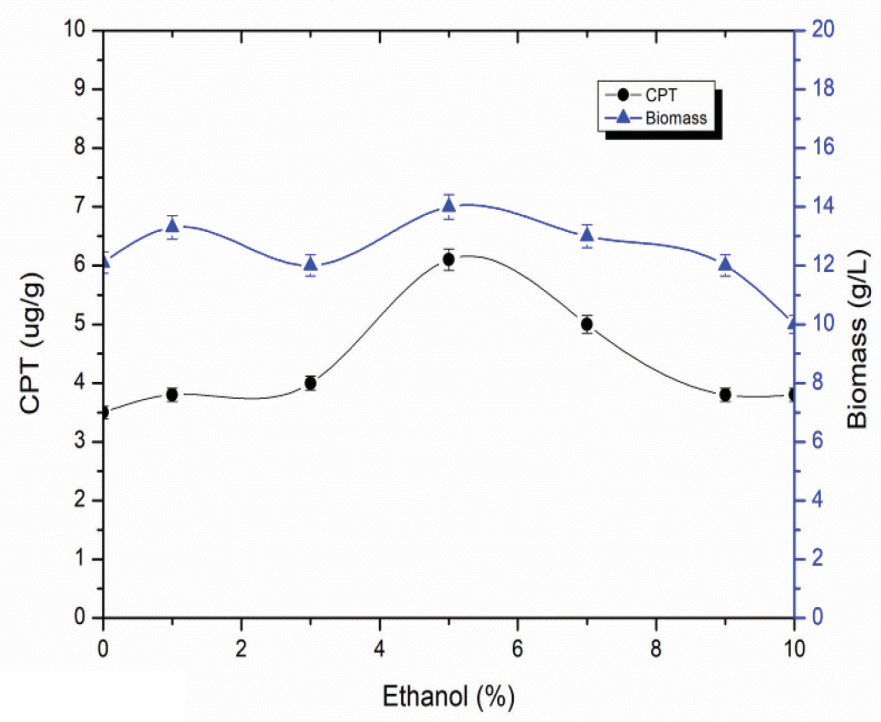

(c)

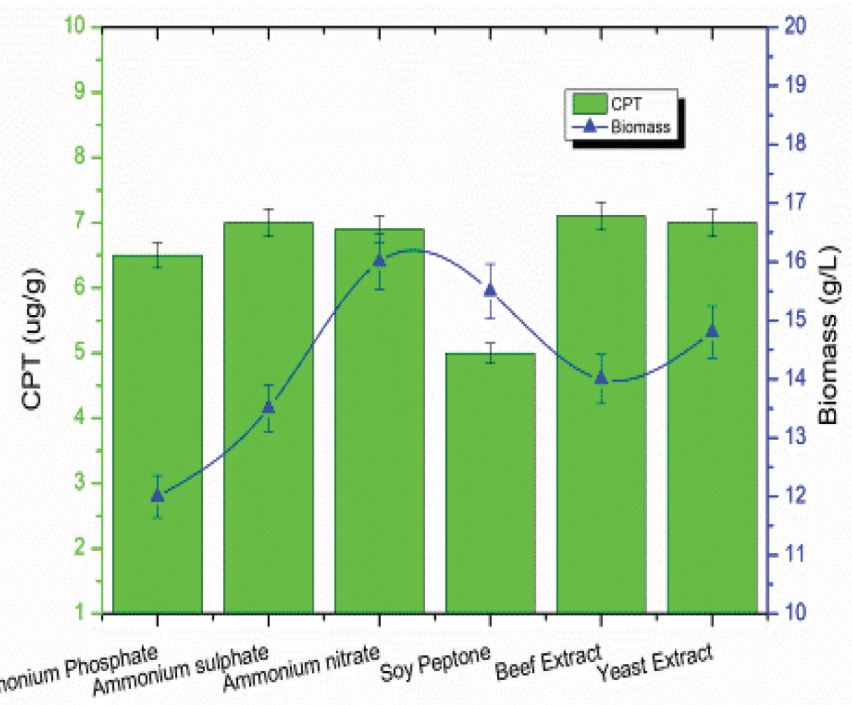

Nitrogen Source (1\%)

(e)

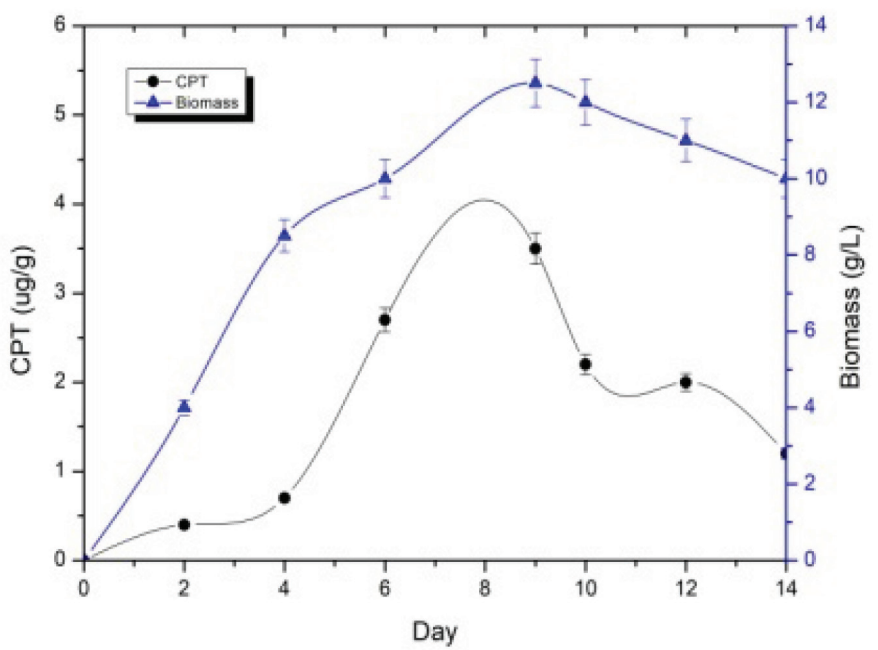

(b)

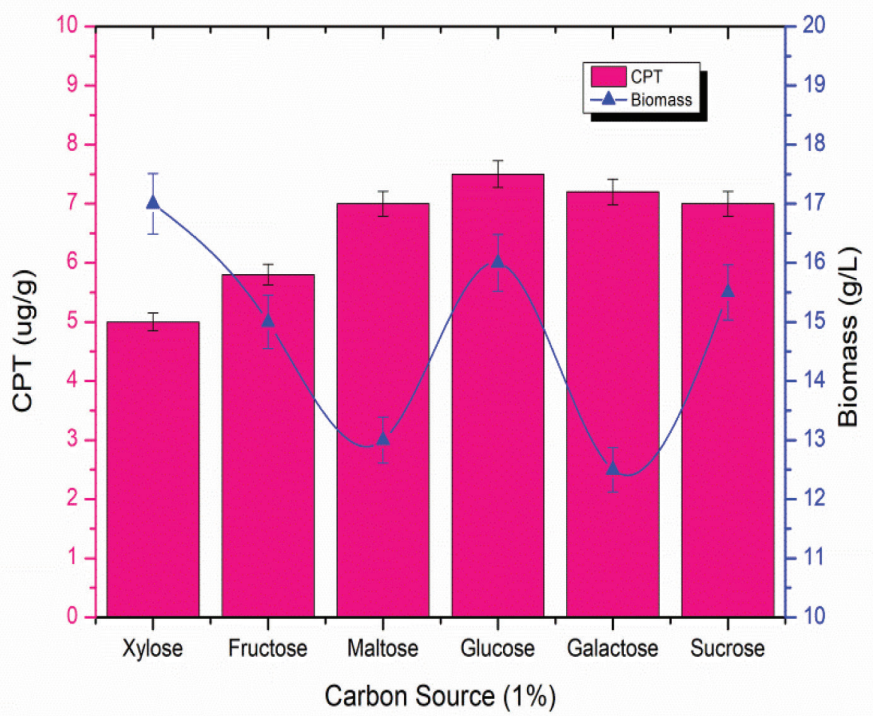

(d)

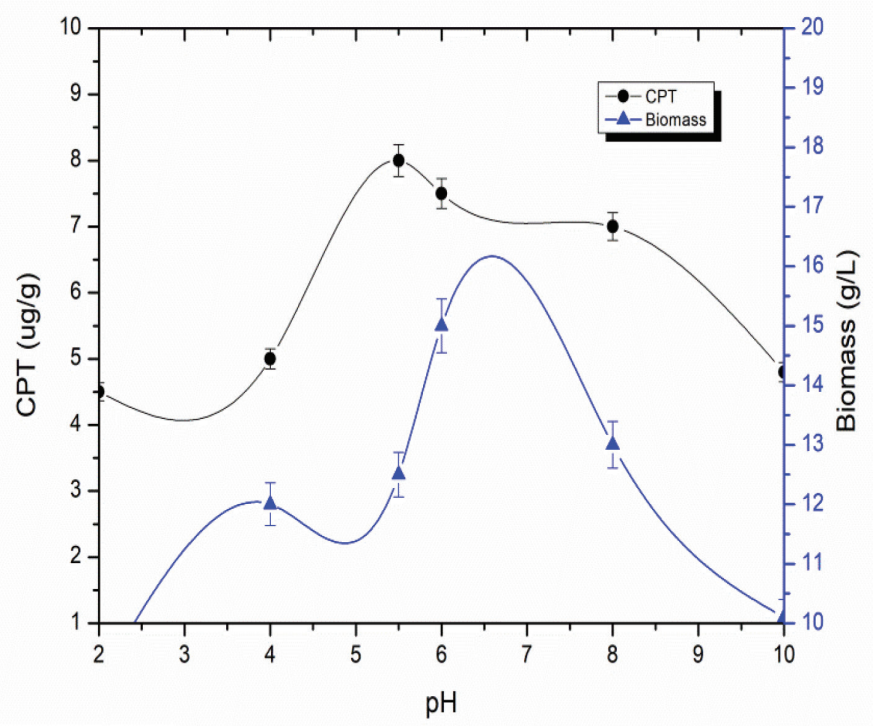

(f) 


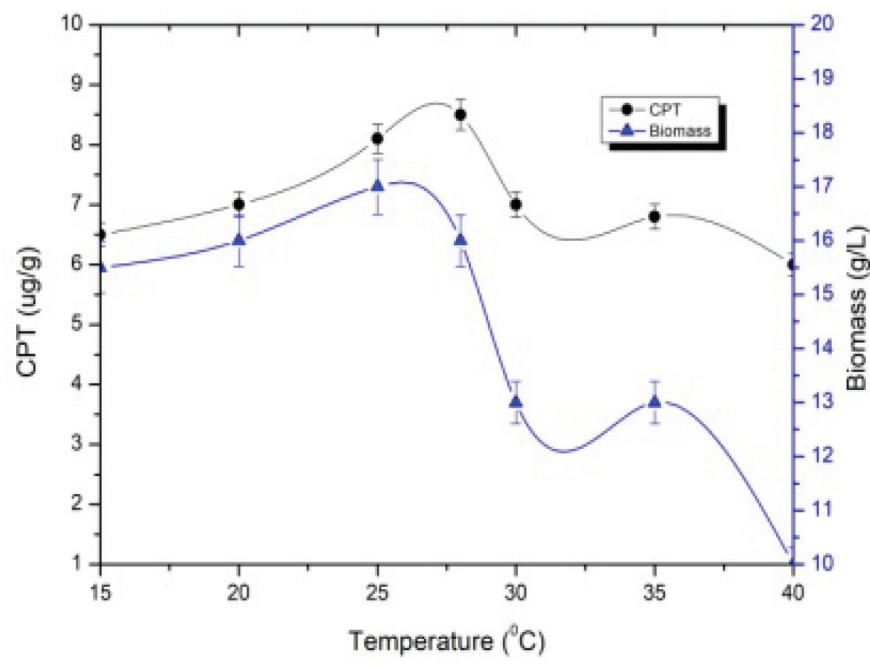

(g)

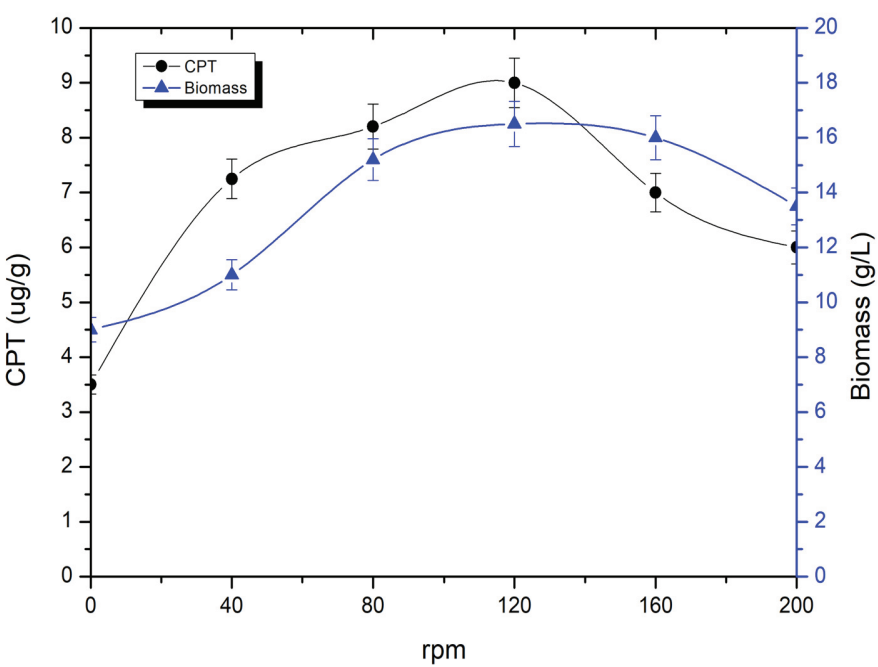

(h)

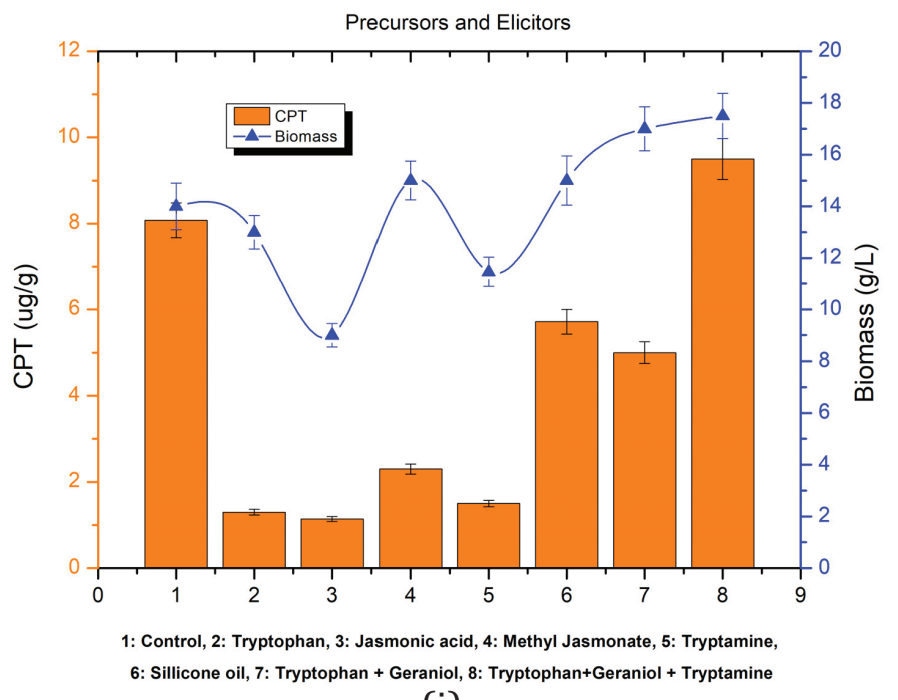

(i)

Figure 4a. Graphs showing optimization of (a) media (b) days (c) ethanol (d) carbon source (e) nitrogen source (f) pH (g) temperature (h) rpm (i) precursors and elicitors using OFAT method.

increased, the growth of the mycelia drastically reduced. At $28^{\circ} \mathrm{C}$, the strain yielded maximum CPT $(8.5 \pm 0.25 \mu \mathrm{g} / \mathrm{ml})$ (Fig. $4 \mathrm{~g})$. The highest CPT $(9 \pm 0.39 \mu \mathrm{g} / \mathrm{ml})$ production and biomass yield $(16.5$ $\pm 0.49 \mathrm{~g} / \mathrm{l})$ were obtained at $120 \mathrm{rpm}$ (Fig. $4 \mathrm{~h}$ ).

\section{Effect of precursors and elicitors}

CPT yield increased substantially by the addition of precursors and elicitors, particularly using the combination of tryptophan, geraniol, and tryptamine $(0.03 \%)$. This combination showed $10.9 \pm 0.32 \mu \mathrm{g} / \mathrm{ml}$ of CPT and $19 \pm 0.57 \mathrm{~g} / 1$ of biomass (Fig. 4i).

\section{Optimization of independent variables using RSM}

Ethanol, glucose, and precursors were selected as independent variables (Table 1). Study of parameters was done at three levels and a total of 17 experiments were carried out as per the variables ranges combinations mentioned in Table 2.
Table 1. Experimental range, level, and code of variables for BBD.

\begin{tabular}{lcccc}
\hline \multirow{2}{*}{ Variables } & \multirow{2}{*}{ Code } & \multicolumn{3}{c}{ Range and Levels } \\
\cline { 3 - 5 } & & $\mathbf{- 1}$ & $\mathbf{0}$ & $+\mathbf{1}$ \\
\hline Ethanol (\%) & $\mathrm{A}$ & 2 & 5 & 8 \\
Glucose (\%) & $\mathrm{B}$ & 0.5 & 1 & 1.5 \\
Precursor (\%) & $\mathrm{C}$ & 0.01 & 0.03 & 0.05 \\
\hline
\end{tabular}

The CPT yield was obtained as per the model equation given below:

$Y(\mu \mathrm{g} / \mathrm{ml})=+11.86-0.0625$ Ethanol -0.0875 Glucose +0.525 Precursors +0.275 Ethanol $\times$ Glucose +0.05 Ethanol

$\times$ Precursors -0.3 Glucose $\times$ Precursors +0.2825 Ethanol $^{2}+$ 0.4825 Glucose $^{2}+0.8075$ Precursors ${ }^{2}$

The biomass yield was determined as per the model equation given below: 
$Z(\mathrm{mg} / \mathrm{l})=+21.02+0.6125$ Ethanol +0.5875 Glucose +0.475 Precursor +0.15 Ethanol $\times$ Glucose +0.575 Ethanol $\times$ Precursor +0.375 Glucose $\times$ Precursor -1.51 Ethanol $^{2}$ 0.41 Glucose $^{2}+0.015$ Precursor $^{2}$

The highest CPT production was obtained from Run no. 14 which consisted of ethanol $(5 \% \mathrm{v} / \mathrm{v})$, glucose $(0.5 \% \mathrm{w} / \mathrm{v})$, and precursors $(0.05 \% \mathrm{w} / \mathrm{v})$. Likewise, the highest biomass production was obtained from run no.5 which consisted of ethanol $(5 \% \mathrm{v} / \mathrm{v})$, glucose $(1.5 \% \mathrm{w} / \mathrm{v})$, and precursors $(0.05 \% \mathrm{w} / \mathrm{v})$

ANOVA for the quadratic model of CPT is shown in Table 3. $F$ value (30.42) is the base of the significance of the model. Chances of occurrence of large $F$-value due to noise are only $0.01 \%$. The validity of the significance of model terms is indicated by $p$ values which are less than 0.05 . Here, C, AB, BC, $\mathrm{A}^{2}, \mathrm{~B}^{2}$, and $\mathrm{C}^{2}$ come under significant model terms. "Lack of Fit $F$ value" of 0.33 specifies a good model. There is an $80.61 \%$ probability that a "Lack of Fit F value" this large could occur due to noise. The multiple correlation coefficient $\left(R^{2}\right)$ was found to be close to $1(0.9751)$. Coefficient of variation $(\mathrm{CV})$ of $1.31 \%$ represents that the experiments are dependable and accurate. Predicted $R^{2}$ of 0.8898 is close to Adjusted $R^{2}$ of 0.9430 .

ANOVA of the quadratic model of fungal biomass is exhibited in Table 4. A, B, C, AB, AC, BC, $\mathrm{A}^{2}$, and $\mathrm{B}^{2}$ are significant model terms. The Model F-value of 387.74 implies the model is significant. Highly reliable and precise experiment performances are verified and denoted here by a low CV of $0.37 \%$. Predicted $\mathrm{R}^{2}$ of 0.9737 is close to Adjusted $\mathrm{R}^{2}$ of 0.9954

The dots close to the diagonal line observed (Fig. 5) indicate the actual values and predicted of CPT. The interdependent interactions between glucose and ethanol, precursors and ethanol,

Table 2. BBD showing experimental and predicted values of the variable for $\mathrm{CPT}$ and biomass yield

\begin{tabular}{|c|c|c|c|c|c|c|c|}
\hline \multirow{2}{*}{ Run order } & \multirow{2}{*}{$\mathbf{A}$} & \multirow{2}{*}{ B } & \multirow{2}{*}{$\mathbf{C}$} & \multicolumn{2}{|c|}{ Estimation of CPT $(\mu \mathrm{g} / \mathrm{ml})$} & \multicolumn{2}{|c|}{ Estimation of biomass (g/l) } \\
\hline & & & & Actual value & Predicted value & Actual value & Predicted value \\
\hline 1 & -1 & 0 & 1 & 13.5 & 13.49 & 18.9 & 18.81 \\
\hline 2 & -1 & -1 & 0 & 13 & 13.05 & 18 & 18.05 \\
\hline 3 & -1 & 0 & -1 & 12.5 & 12.54 & 19 & 19.01 \\
\hline 4 & 0 & 0 & 0 & 11.8 & 11.86 & 21 & 21.02 \\
\hline 5 & 0 & 1 & 1 & 13.2 & 13.29 & 22 & 22.06 \\
\hline 6 & 0 & 1 & -1 & 12.8 & 12.84 & 20.4 & 20.36 \\
\hline 7 & 1 & 0 & -1 & 12.3 & 12.31 & 19 & 19.09 \\
\hline 8 & 0 & 0 & 0 & 12.2 & 11.86 & 21 & 21.02 \\
\hline 9 & 1 & 0 & 1 & 13.5 & 13.46 & 21.2 & 21.19 \\
\hline 10 & 1 & 1 & 0 & 12.8 & 12.75 & 20.5 & 20.45 \\
\hline 11 & -1 & 1 & 0 & 12.4 & 12.32 & 18.9 & 18.92 \\
\hline 12 & 1 & -1 & 0 & 12.3 & 12.38 & 19 & 18.97 \\
\hline 13 & 0 & -1 & -1 & 12.5 & 12.41 & 20 & 19.94 \\
\hline 14 & 0 & -1 & 1 & 14.1 & 14.06 & 20.1 & 20.14 \\
\hline 15 & 0 & 0 & 0 & 11.7 & 11.86 & 21.1 & 21.02 \\
\hline 16 & 0 & 0 & 0 & 11.8 & 11.86 & 21 & 21.02 \\
\hline 17 & 0 & 0 & 0 & 11.8 & 11.86 & 21 & 21.02 \\
\hline
\end{tabular}

Table 3. ANOVA for CPT production.

\begin{tabular}{|c|c|c|c|c|c|c|}
\hline Source & Sum of squares & df & Mean square & $F$-value & \multicolumn{2}{|c|}{$p$-value } \\
\hline Model & 7.41 & 9 & 0.8234 & 30.42 & $<0.0001$ & Significant \\
\hline A-Ethanol & 0.0312 & 1 & 0.0312 & 1.15 & 0.3183 & \\
\hline B-Glucose & 0.0612 & 1 & 0.0612 & 2.26 & 0.1762 & \\
\hline C-Precursors & 2.20 & 1 & 2.20 & 81.45 & $<0.0001$ & \\
\hline $\mathrm{AB}$ & 0.3025 & 1 & 0.3025 & 11.17 & 0.0124 & \\
\hline $\mathrm{AC}$ & 0.0100 & 1 & 0.0100 & 0.3694 & 0.5625 & \\
\hline $\mathrm{BC}$ & 0.3600 & 1 & 0.3600 & 13.30 & 0.0082 & \\
\hline $\mathrm{A}^{2}$ & 0.3360 & 1 & 0.3360 & 12.41 & 0.0097 & \\
\hline $\mathrm{B}^{2}$ & 0.9802 & 1 & 0.9802 & 36.21 & 0.0005 & \\
\hline $\mathrm{C}^{2}$ & 2.75 & 1 & 2.75 & 10142 & $<00001$ & \\
\hline Residual & 0.1895 & 7 & 0.0271 & 101.42 & 0.0001 & \\
\hline Lack of fit & 0.0375 & 3 & 0.0125 & & & Not significant \\
\hline Pure error & 0.1520 & 4 & 0.0380 & 0.3289 & 0.8061 & \\
\hline Cor total & 7.60 & 16 & & & & \\
\hline
\end{tabular}

$R^{2}-0.9751$, Adj $R^{2}-0.9430$, Predicted $R^{2}-0.8898, \mathrm{CV}-1.31 \%$, Adeq precision—17.4536, Df-degree of freedom, Significant $-p \leq 0.05$, Non-significant— $p>0.05$. 
Table 4. ANOVA for biomass production.

\begin{tabular}{|c|c|c|c|c|c|c|}
\hline Source & Sum of squares & df & Mean square & $F$-value & \multicolumn{2}{|c|}{$p$-value } \\
\hline Model & 20.19 & 9 & 2.24 & 387.74 & $<0.0001$ & Significant \\
\hline A- Ethanol & 3.00 & 1 & 3.00 & 518.73 & $<0.0001$ & \\
\hline B-Glucose & 2.76 & 1 & 2.76 & 477.25 & $<0.0001$ & \\
\hline C-Precursor & 1.80 & 1 & 1.80 & 311.98 & $<0.0001$ & \\
\hline $\mathrm{AB}$ & 0.0900 & 1 & 0.0900 & 15.56 & 0.0056 & \\
\hline $\mathrm{AC}$ & 1.32 & 1 & 1.32 & 228.58 & $<0.0001$ & \\
\hline $\mathrm{BC}$ & 0.5625 & 1 & 0.5625 & 97.22 & $<0.0001$ & \\
\hline $\mathrm{A}^{2}$ & 9.60 & 1 & 9.60 & 1659.33 & $<0.0001$ & \\
\hline $\mathrm{B}^{2}$ & 0.7078 & 1 & 0.7078 & 122.33 & $<0.0001$ & \\
\hline $\mathrm{C}^{2}$ & 0.0009 & 1 & 0.0009 & 01637 & 06078 & \\
\hline Residual & 0.0405 & 7 & 0.0058 & & 0.0970 & \\
\hline Lack of fit & 0.0325 & 3 & 0.0108 & & & not significant \\
\hline Pure error & 0.0080 & 4 & م & 5.42 & 0.0681 & \\
\hline Cor total & 20.23 & 16 & & & & \\
\hline
\end{tabular}

and precursors and glucose for enhanced CPT production are shown in Figure 6a-c. Three-dimensional graphs results showed a significant effect of precursors on CPT production. The actual and predicted values of biomass production were observed to be close to the diagonal line (Fig. 7). The interdependent interactions between glucose and ethanol, precursors and ethanol, and precursors and glucose for enhanced biomass yield are shown in Figure 8a-c. Three-dimensional graphs results showed a significant effect of glucose, precursors, and ethanol on biomass yield. Further, the CPT production and biomass yield were compared with the theoretical values by carrying out the experiments under optimized conditions. Experimental values were found close with the theoretical values.

\section{DISCUSSION}

Over the last few decades, endophytic fungi have created immense attention among worldwide researchers for distinct secondary metabolites of plants (Musavi et al., 2015). In this regard, several diversified bioactive components have been isolated and characterized as therapeutics (Shweta et al., 2010). However, studies revealing the potential of endophytic fungi as promising resources of such valuable constituents are scarce. In view of this, in the present investigation, we had undertaken a significant attempt not only to isolate endophytic fungus from C. fragrans but also optimizing the varied parameters to enhance the production of CPT from it. In this study, the endophytic fungus F. solani strain ATLOY-8 was successfully isolated from C. fragrans using morphological and molecular characterization tools. However, similar endophytic fungus from the different plant was isolated earlier from Venugopalan et al. (2016) too. The potentiality of many endophytic fungi has been explored and subjected for the synthesis of CPT outside their plant hosts. The reports of methods for augmentation of CPT are on record already (Musavi et al., 2015; Rehman et al., 2009). But still, economic viability and better yield of CPT by endophytic fungi is a matter of great concern and challenge.

Methods of conventional optimization techniques for monocultures and techniques and procedures of mixed fermentation

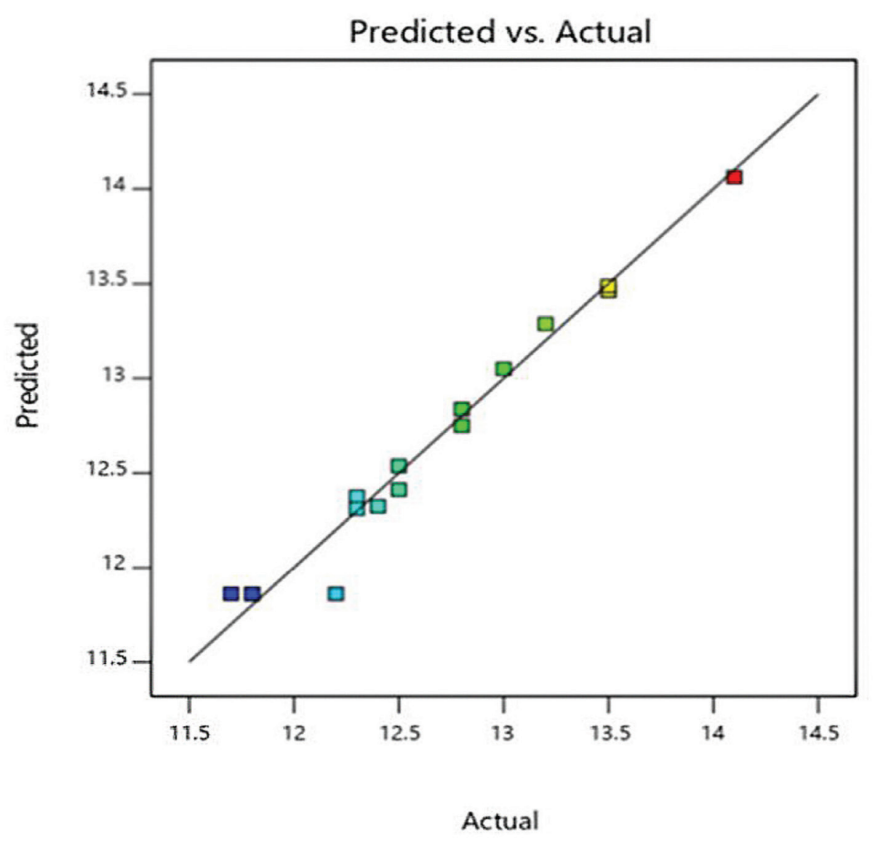

Figure 5. Actual values vs predicted values for biomass yield residing close to the diagonal line.

gave adequate knowledge of the highly prompting parameters and their optimum values (Bhalkar et al., 2016). Medium constituents and culture parameters in optimization play an important role in the enhancement of metabolite productivity as well as microbial biomass yield in submerged fermentation. Among various media tested, strain ATLOY-8 showed maximum production of CPT and biomass yield in YESD medium. Likewise, the incubation period affected the production and biomass significantly. Maximum CPT production and biomass yield were estimated on day 9, while the production and yield decreased gradually afterward. The adding of absolute ethanol at varied concentrations affected the CPT and biomass yield from strain ATLOY-8. Maximum production of CPT and biomass 


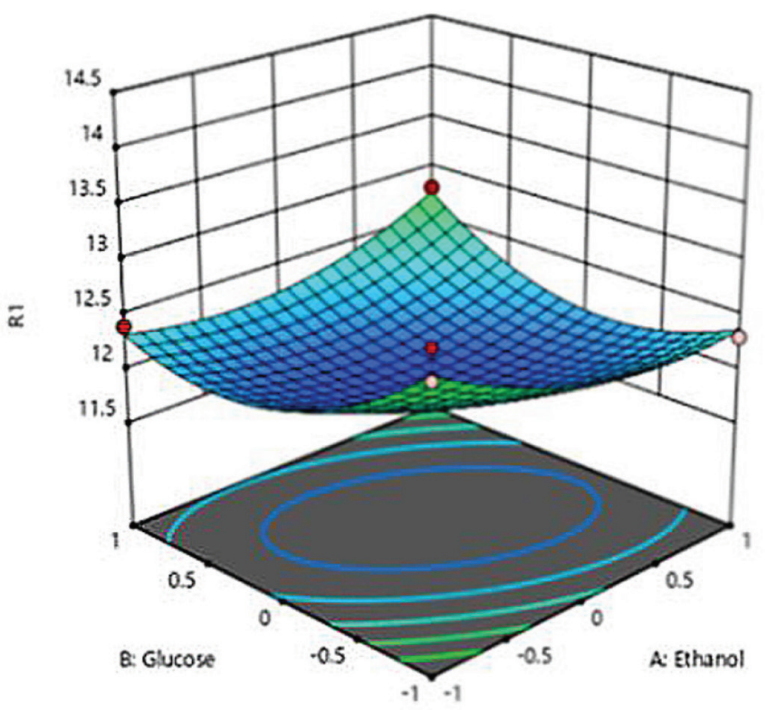

(a)

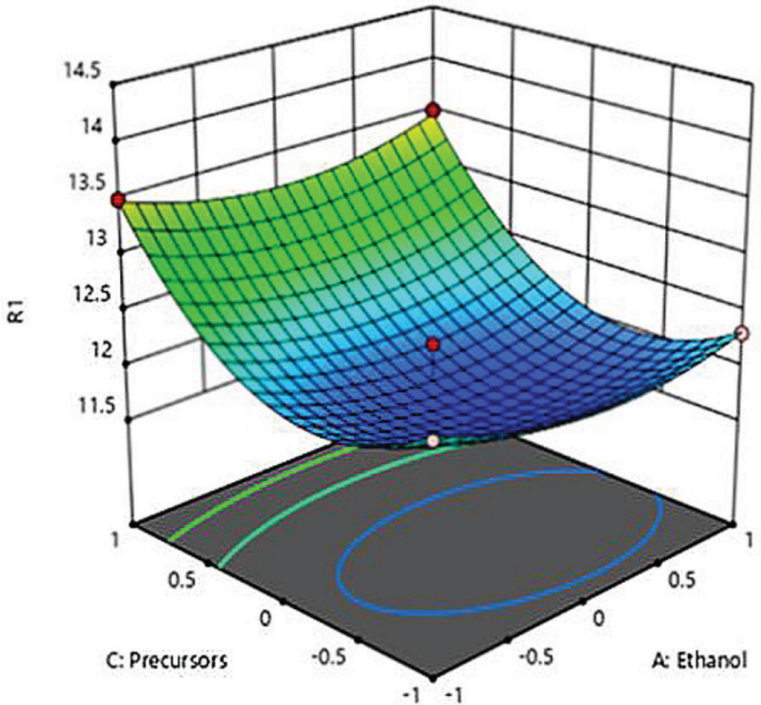

(b)

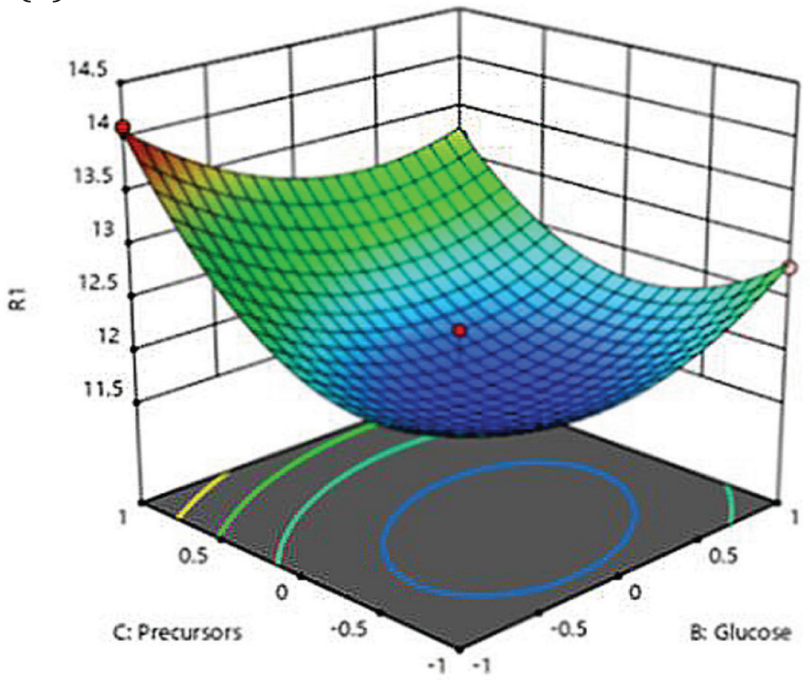

(c)

Figure 6. 3D response plot for CPT production showing the interaction between (a) glucose and ethanol (b) precursors and ethanol, and (c) precursors and glucose.

was estimated in the presence of $5 \%(\mathrm{v} / \mathrm{v})$ of ethanol. This might be due to the elicitor as well as energy source role of ethanol for this isolate, thereby exhibiting high CPT and biomass production. Similar observation was reported by Venugopalan and Srivastava (2015) who estimated the significant increment in the production of CPT due to the incorporation of absolute ethanol into the medium. Among different carbon sources tested, strain ATLOY- 8 showed a maximum yield of CPT and biomass in the presence of glucose $(1 \%$ $\mathrm{w} / \mathrm{v})$, followed by galactose, maltose, sucrose, fructose, and xylose. In like manner, the incorporation of beef extract $(1 \% \mathrm{w} / \mathrm{v})$ into the basal medium revealed a high yield of CPT and biomass.

The $\mathrm{pH}$ of the medium is one of the crucial factors affecting the growth and metabolite production from the microorganisms. In the present study, strain ATLOY-8 showed maximum CPT and biomass production at $\mathrm{pH}$ 6.0. Acidic $\mathrm{pH}$ reduced the growth of mycelia significantly, while in the alkaline $\mathrm{pH}$, the growth of mycelia was very slow. The report agrees slightly with the findings of Venugopalan et al. (2016) who determined the enhanced yield of CPT at $\mathrm{pH}$ 6.0. Temperature is a very important physical factor for the growth of mycelia in the specific medium. In this study, strain ATLOY- 8 showed maximum biomass production at $25^{\circ} \mathrm{C}$. Further increment in the incubation temperature reduced the growth and metabolite production drastically. This might be due to the reason that the enzyme synthesizing CPT in this very strain would be the most active at this temperature. Surprisingly, the findings of our study do not favor the outcome of Venugopalan et al. (2016) who depicted gradual improvement in CPT yield with an increase in temperature. Likewise, the trend in the enhancement of CPT yield from endophytic fungus with increased temperature was observed by $\mathrm{Pu}$ et al. (2013) too.

Agitation speed is another critical factor affecting the metabolite production and growth of microbes. In this context, strain ATLOY-8 demonstrated a maximum yield of CPT and 


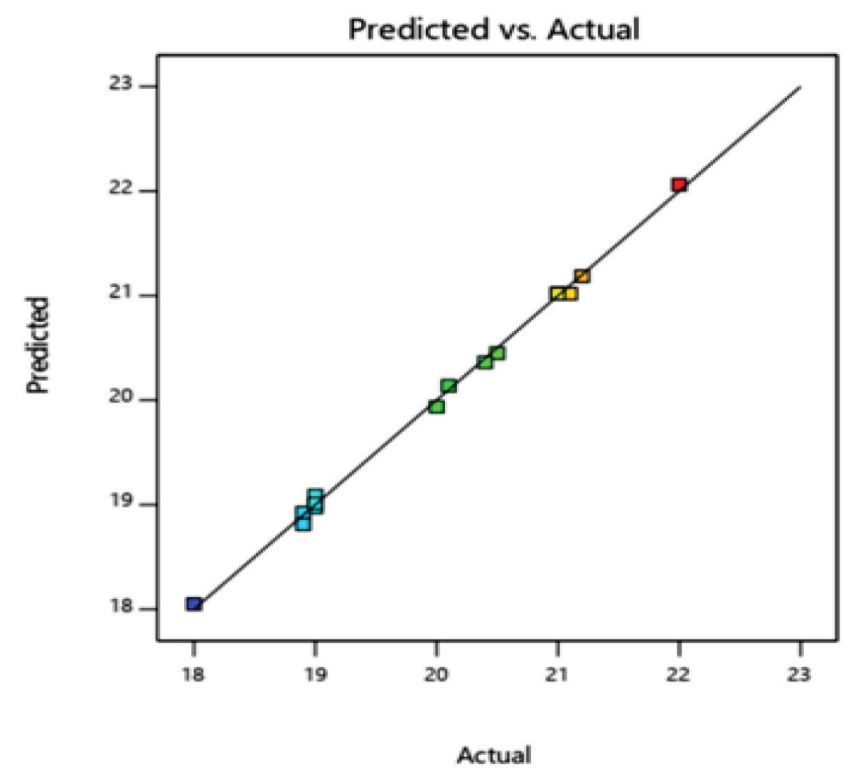

Figure 7. Actual values vs predicted values for biomass yield residing close to the diagonal line. biomass at $120 \mathrm{rpm}$. Further increased agitation speed reduced productivity. This might be because of the shear stress causing a loss in cellular viability, thereby affecting the metabolite production. However, the lower production of CPT and biomass yield from the very strain at low agitation speed might be because of the limitations in mass transfer (Venugopalan et al., 2016). The exogenous addition of precursors and elicitors, particularly the combination of tryptophan, geraniol, and tryptamine exhibited the maximum productivity of CPT and biomass. The reduction in CPT and biomass production upon the incorporation of other precursors and elicitors could be because of the cellular toxicity at the particular doses provided. More or less similar outcomes of the positive influence of elicitor and precursor addition on CPT production from endophytic fungus have been reported by $\mathrm{Pu}$ et al. (2013).

The traditional tools of optimization, i.e., OFAT method is being avoided nowadays because of its time consuming and low accuracy approach (Aarti et al., 2018; Khusro et al., 2017). Response surface methodology is a technique that combines mathematical as well as statistical methods and it can undoubtedly be a promising alternative to the OFAT method. As a matter of

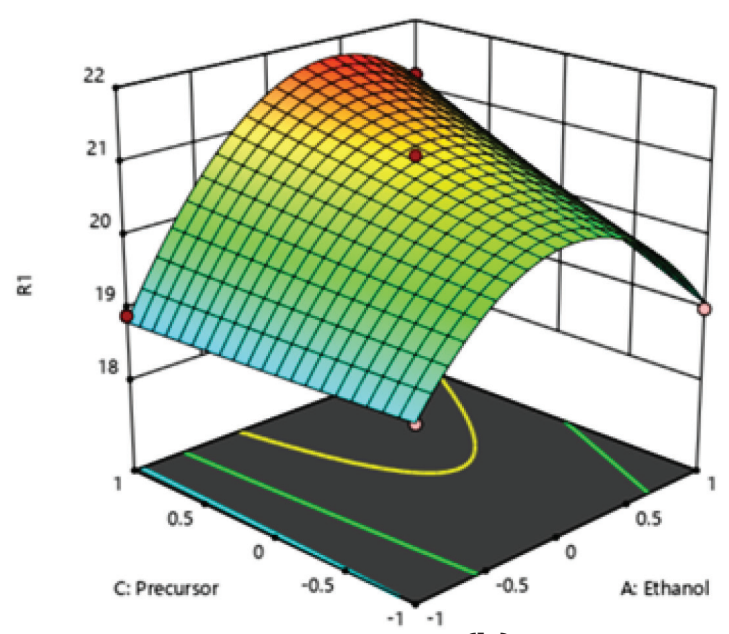

(b)

(a)

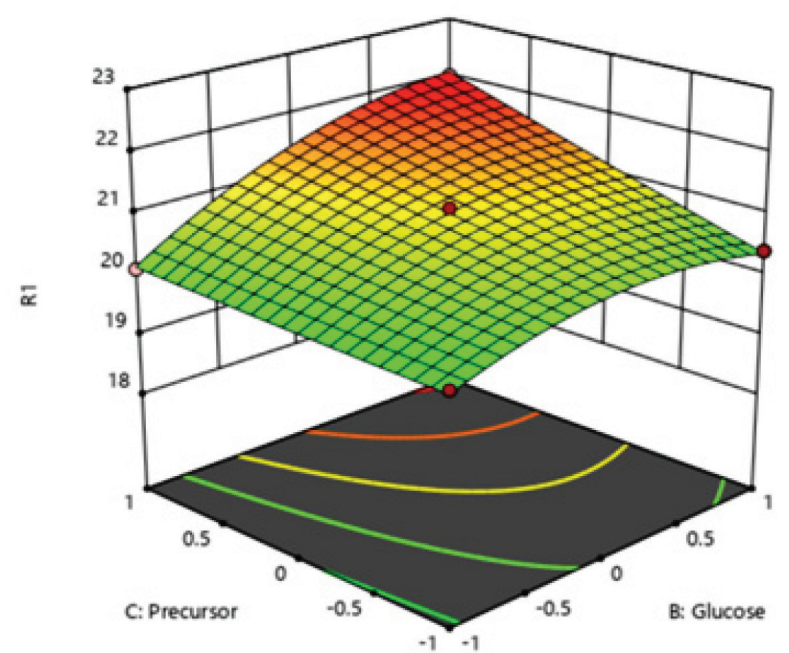

(c)

Figure 8. 3D response plot for biomass production showing the interaction between (a) ethanol and glucose (b) precursors and ethanol, and (c) precursors and glucose. 
fact, RSM analyzes the interdependent relationships between the dependent and independent variable and help carrying out a minimum number of experiments with high accuracy (Aarti et al., 2017). In the present context, the BBD of RSM led to the enhanced CPT production $(14.1 \mu \mathrm{g} / \mathrm{g})$ and biomass yield $(22.2 \mathrm{~g} / \mathrm{l})$ in the medium containing ethanol $(5 \% \mathrm{v} / \mathrm{v})$, glucose $(0.5 \% \mathrm{w} / \mathrm{v})$, and precursor $(0.05 \% \mathrm{w} / \mathrm{v})$. ANOVA, as well as $3 \mathrm{D}$ graphs of CPT production and biomass yield, clearly showed the significant $(p<$ 0.05) effect of precursors in order to enhance the responses.

Contour plots demonstrate insignificant interaction between two controlled variables. The slight variations between experimental and predicted response values were because of the determination coefficients (Khusro et al., 2018). Previously, Bhalkar et al. (2016) successfully enhanced CPT yield from endophytic fungus using statistical tools, suggesting the pivotal role of statistical tools based optimization of metabolites from endophytes.

\section{CONCLUSION}

In brief, the CPT producing endophytic fungus $F$. solani strain ATLOY-8 was isolated from $C$. fragrans using morphological and molecular characterization tools. HPLC and ESI-Q-TOF MS analysis showed the notable production of CPT from strain ATLOY-8. The promising factors selected through OFAT method were further optimized using BBD which showed enhanced CPT $(14.1 \mu \mathrm{g} / \mathrm{g})$ and biomass $(22.0 \mathrm{~g} / \mathrm{l})$ yield in the production medium containing ethanol $(5 \% \mathrm{v} / \mathrm{v})$, glucose $(0.5 \% \mathrm{w} / \mathrm{v})$, and precursor $(0.05 \% \mathrm{w} / \mathrm{v})$. ANOVA, as well as $3 \mathrm{D}$ graphs of CPT production and biomass yield, showed the significant $(p<0.05)$ effect of precursors toward the enhancement of responses. Experimental data of response showed closeness with the predicted data.

\section{CONFLICT OF INTEREST}

Authors declare that they have no conflicts of interest.

\section{REFERENCES}

Aarti C, Khusro A, Agastian P. Goat dung as a feedstock for hyper-production of amylase from Glutamicibacter arilaitensis strain ALA4. Bioresour Bioprocess, 2017; 4:43.

Aarti C, Khusro A, Agastian P. Carboxymethyl cellulase production optimization from Glutamicibacter arilaitensis strain ALA4 and its application in lignocellulosic waste biomass saccharification. Prep Biochem Biotechnol, 2018; 48:853-66.

Backlund M, Oxelman B, Bremer B. Phylogenetic relationships within the Gentianales based on $\mathrm{ndhF}$ and $\mathrm{rbcL}$ sequences, with particular reference to the Loganiaceae. Am J Bot, 2000; 87:1029-43.

Bhalkar BN, Patil SM, Govindwar SP. Camptothecine production by mixed fermentation of two endophytic fungi from Nothapodytes nimmoniana. Fungal Biol, 2016; 120:873-83.

Barnett HL, Hunter BB. Illustrated General of Imperfect fungi. 4h edition, The American Phytopathological Society, Minnesota, 1998.

Bocchini DA, Alves-Prado HF, Baida LC, Roberto IC, Gomes E, Da Silva R. Optimization of Xylanase production by Bacillus circulans D1 in submerged fermentation using response surface methodology. Process Biochem, 2002; 38:727-31.

Demain AL, Vaishnav P. Natural products for cancer chemotherapy. Microb Biotechnol, 2011; 4:687-99.

Hallmann J, Berg, G, Schulz B. Isolation procedure for endophytic microorganisms. In: Boyle, Ch JC, Sieber TN, Schulz BJE (eds.). Microbial root endophytes, Springer, Berlin, Heidelberg, pp 299 305, 2006

Hilu KW, Borsch T, Müller K, Soltis DE, Soltis PS, Savolainen V, Chase MW, Powell MP, Alice LA, Evans R, Sauquet H, Neinhuis C, Slotta TA, Rohwer JG, Campbell CS, Chatrou LW. Angiosperm phylogeny based on matK sequence information. Am J Bot, 2003; 90:1758-76.

Hsiang YH, Liu LF. Identification of mammalian DNA Topoisomerase I as an intracellular target of the anticancer drug camptothecin. Cancer Res, 1988; 48:1722-6.

Khan S, Karnat NM, Darshan S. India's foundation for revitalization of local health traditions, pioneering In situ conservation strategies for medicinal plants and local cultures. Herbalgram, 2005; 68:3445 .

Khusro A, Aarti C, Dusthackeer A, Agastian P. Enhancement of anti-tubercular activity and biomass of fermented food associated Staphylococcus hominis strain MANF2 using Taguchi orthogonal array and Box-Behnken design. Microb Pathog, 2018; 120:8-18.

Khusro A, Aarti C. Molecular identification of newly isolated Bacillus strains from poultry farm and optimization of process parameters for enhanced production of extracellular amylase using OFAT method. Res J Microbiol, 2015; 10:393-420

Khusro A, Barathikannan K, Aarti C, Agastian P. Optimization of thermo-alkali stable amylase production and biomass yield from Bacillus sp. under submerged cultivation. Fermentation, 2017; 3:1-19.

Khusro A, Kaliyan B, Al-Dhabi NA, Arasu MV, Agastian P. Statistical optimization of thermo- alkali stable xylansae production from Bacillus tequilensis strain ARMATI. Electron J Biotechnol, 2016; 22:16-25.

Kjeldsen E, Sevjestrup JQ, Gromova II, Alsner J, Westergaard O. Camptothecin inhibits both the cleavage and religation reactions of eukaryotic DNA Topoisomerase I. J Mol Biol, 1992; 228:1025-30.

Lorence, A, Nessler C L. Molecules of interest camptothecin, over four decades of surprising findings. Phytochemistry, 2004; 65:273549.

Musavi SF, Dhavale A, Balakrishnan RM, 2015. Optimization and kinetic modeling of cell500 associated camptothecin production from an endophytic Fusarium oxysporum NFX06. Prep Biochem Biotechnol, $2015 ; 45: 158-72$

$\mathrm{Pu}$ X, Qu X, Chen F, Bao J, Zhang G, Luo Y. Camptothecinproducing endophytic fungus Trichoderma atroviride LY357: isolation, identification, and fermentation conditions optimization for camptothecin production. Appl Microbiol Biotechnol, 2013; 97:9365-75.

Raveendran VV. Camptothecin-discovery, clinical perspectives and biotechnology. Nat Prod Chem Res, 2015; 3:175.

Rehman S, Shawl AS, Kour A, Sultan P, Ahmad K, Khajuria R, Qazi GN. Comparative studies and identification of camptothecin produced by an endophyte at shake flask and bioreactor. Nat Prod Res, 2009; 23:1050-7.

Shweta S, Zuehlke BT, Ramesha V, Priti P, Mohana Kumar G, Ravikanth M, Spiteller R, Vasudeva R, Uma Shaanker R. Endophytic fungal strains of Fusarium solani, from Apodytes dimidiata E. Mey. ex Arn (Icacinaceae) produce camptothecin, 10-hydroxycamptothecin and 9-methoxycamptothecin. Phytochemistry, 2010; 71:117-22.

Shwetha S. Gurumurthy B, Ravikanth G, Ramanan U, Shivanna M. Endophytic fungi from Miquelia dentata Bedd, produce the anti-cancer alkaloid, camptothecine. Phytomedicine, 2013; 20:337-42.

Thomas CJ, Rahier NJ, Hecht SM. Camptothecin: current perspectives. Bioorgan Med Chem, 2004; 12: 1585-604.

VanderMolen KM, Raja HA, El-Elimat T, Oberlies NH Evaluation of culture media for the production of secondary metabolites in a natural products screening program. AMB Express, 2013; 3:1-7.

Venugopalan A, Srivastava S. Enhanced camptothecin production by ethanol addition in the suspension culture of the endophyte, Fusarium solani. Bioresour Technol, 2015; 188:251-7. 
Venugopalan A, Potunuru UR, Dixit M, Srivastava S. Effect of fermentation parameters, elicitors and precursors on camptothecin production from the endophyte Fusarium solani. Bioresour Technol, 2016; 213:311-8

White T, Bruns T, Lee S, Taylor J. Amplification and direct sequencing of fungal ribosomal RNA Genes for phylogenetics. In: Innis, M, Gelfand D, Sanisky J, White T (eds.). PCR protocols: a guide to methods and applications, Academic Press, San Diego, pp 315-22, 1990.
How to cite this article:

Clarance P, Khusro A, Lalitha J, Sales J, Paul A. Optimization of camptothecin production and biomass yield from endophytic fungus Fusarium solani strain ATLOY-8. J Appl Pharm Sci, 2019; 9(10):035-046. 